\section{BUILD YOUR IMPLANT BUSINESS}

The Implant Hub is delighted to announce that access to its content is now available to all dentists, with a few exclusive areas remaining accessible to members only.

A site where you can gain valuable insight into growing your dental business, The Implant Hub delivers exclusive support and advice in implant dentistry through articles and blogs to Google Hangouts, as well as LIVE QCtAs from top coaches.

These include newly appointed Branding Coach, Les Jones, Creative Director of Practice Plan, and Financial Coach, Johnny Minford of Minford Accountants, alongside Chris Barrow (Business Coach), Mark Oborn (Marketing Coach) and Dr Nav Ropra (Inspirational Coach).

Recently added content includes:

- Les Jones on 'How to boost your brand and maximise your dental treatment leads'

- 'Why Facebook matters for your implant marketing' by Chris Barrow

- Mark Oborn writing about 'How to increase your dental sales with automated emails'.

In addition, video content will be a significant focus on the website for 2016. What's more, white papers are available to The Implant Hub members, including Chris Barrow's offering on 'Growing your implant practice by understanding the financials' and Mark Oborn focusing on 'The best 7 3/4 tips you'll ever see to using online marketing to help more local people eat, smile and chew with confidence'.

To see for yourself what The Implant Hub has to offer your implant dentistry business, visit www.theimplanthub.com.

\section{FREE CPD ON CARING FOR PATIENTS WITH DENTURES}

By 2050 our global population of those aged 60 years or older is expected to more than double to two billion people. Statistics reveal the older you are the more likely you are to be edentulous. Research shows that denture patients are using a mix of up to 14 different methods to clean their dentures ranging from soap and water to bleach. In one study only $12 \%$ of subjects had clean dentures. ${ }^{1}$

To provide further insight for dental professionals, GSK, manufacturers of Poligrip, have launched a distance learner module. The module content examines the impact of an ageing population, the effects of tooth loss and dentures for patients and the role of denture fixatives and cleansers.

The module is available at www.gsk-dentalprofessionals. co.uk for easy access and is free to complete. Completion can contribute up to 1.5 hours towards verifiable CPD.

1. Dikbas I, Koksal T, Calikkocaoglu S. Investigation of the cleanliness of dentures in a university hospital. Int J Prosthodont 2006; 19: 194-298

\title{
FIRST UK ONLINE JAW TREATMENT RESOURCE FOR PATIENTS
}

The British Orthodontic Society (BOS) has launched the first UK online resource for patients considering orthognathic treatment, a dual procedure combining jaw surgery and orthodontics.

Each year it's estimated that around 3,000 people have this treatment with an overwhelming 90\% saying it has dramatically improved their health and wellbeing.

Orthognathic treatment is used in the treatment of adults with developmental conditions, including cleft palate, patients who have difficulty in breathing while asleep and patients with difficulties in eating. Carried out jointly by an oral and maxillofacial surgeon dually qualified in medicine and dentistry - and an orthodontist, its purpose is to move the teeth and jaws into positions that are more balanced, functional and healthy, enabling patients to eat and breathe

without restriction.

\section{THE NEXT CHAPTER FOR IMPLANT DENTISTRY}

ImplantPilot is a revolutionary surgical guide that is set to change implant dentistry as we know it. Safe, accurate, and cost effective this unique system reduces the stress of placing implants for the surgeon and patient alike. For the first time ever patients are given the option of keyhole surgery with no stitches, reduced time in the chair and faster recovery times when compared to traditional implant placement.

With ImplantPilot, a CT scan of the patient and STL scans taken in the laboratory are merged with the aid of a Lego block fiducial marker and integrated into a software planning system operated by 2ingis. The implants are then digitally placed. The ImplantPilot guide is fabricated by 3D printing from autoclaveable sintered cobalt chrome forming an open framework which allows the implant site to be viewed and irrigated. A handpiece with struts fits into the guide holes on either side of the implant site enabling position, angulation and even depth to be guaranteed. The implants and drill sequence are concisely prescribed yourjawsurgery.com is a comprehensive online resource divided into four clear sections including:

- Patient Journey: Ten films covering each step of the treatment journey

- Patient Stories: Fourteen films with real patients talking about their story and their treatment. Includes 'before' and 'after' photos

- Your Surgery Explained:

Thirteen animated films clearly showing the different types of surgery

- Other Resources: Advice sheets and links to other websites.

The resource was two years in the making, led by consultant orthodontists Caroline McCarthy and Lindsay Winchester. Filming took place at Queen Victoria Hospital in East Grinstead and Sheffield Dental School.

www.yourjawsurgery.com

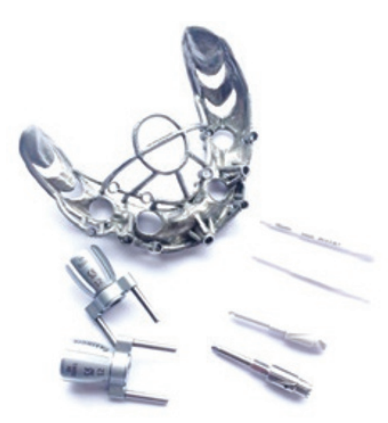

along with the guide facilitating placement by a simple stepwise procedure.

There is no catch, which is why this revolutionary, minimally invasive, time efficient implant option is proving to be so popular. It's the next chapter for implant dentistry.

It's suitable for all implant systems; the software cost is $£ 600$ annually and the drill kits start from $£ 2,200$ + VAT. There is training and even a starter package, 'The Launchpad', to get you started without any capital investment.

You can find out more about ImplantPilot by calling 01274296842 to discuss any questions you may have, or visit www.implantpilot.co.uk. 